\title{
Loss of gene function and evolution of human phenotypes
}

\author{
Hye Ji Oh, Dongjin Choi, Chul Jun Goh E Yoonsoo Hahn* \\ Department of Life Science, Research Center for Biomolecules and Biosystems, Chung-Ang University, Seoul 156-756, Korea
}

\begin{abstract}
Humans have acquired many distinct evolutionary traits after the human-chimpanzee divergence. These phenotypes have resulted from genetic changes that occurred in the human genome and were retained by natural selection. Comparative primate genome analyses reveal that loss-of-function mutations are common in the human genome. Some of these gene inactivation events were revealed to be associated with the emergence of advantageous phenotypes and were therefore positively selected and fixed in modem humans (the "less-ismore" hypothesis). Representative cases of human gene inactivation and their functional implications are presented in this review. Functional studies of additional inactive genes will provide insight into the molecular mechanisms underlying acquisition of various human-specific traits. [BMB Reports 2015; 48(7): 373-379]
\end{abstract}

\section{INTRODUCTION}

Humans diverged from the chimpanzee lineage approximately 5-7 million years ago (MYA) (1-3). Humans have many specific traits compared with closely related apes that must have resulted from genetic modifications acquired during evolution (2). For example, the human FOXP2 gene shows accelerated amino acid sequence substitutions during human evolution and may have played an important role in language and speech evolution by altering transcription of genes responsible for the development of the central nervous system $(4,5)$. Human accelerated region 1 noncoding RNA shows accelerated nucleotide sequence substitutions in the human lineage and is highly expressed in human neocortex, possibly playing a role in the evolution of human brain development $(6,7)$.

Sequence comparisons of complete genomes of human and related primates has enabled the large-scale identification of genetic modifications in the human lineage, including accelerated sequence substitutions, novel transcript isoforms, and

${ }^{*}$ Corresponding author. Tel: +82-2-820-5812; Fax: +82-2-8255206; E-mail: hahny@cau.ac.kr

http://dx.doi.org/10.5483/BMBRep.2015.48.7.073

Received 11 April 2015

Keywords: Human evolution, Gene inactivation, Pseudogene, Lessis-more hypothesis acquisitions of posttranslational modification sites (6, 8-14). Molecular functions of most of these changes and their associated human-specific phenotypes are not yet established.

According to the "less-is-more" hypothesis, loss of gene function can be implicated in the evolution of human-specific traits (15). In general, loss of gene function by disrupting mutations would be deleterious to individual fitness, and purifying selection would remove the mutant allele from the population. However, environmental or behavioral changes of an organism may relax the selection constraints on a gene, which could then accumulate disrupted mutations without loss of fitness. Under certain circumstances, the absence of intact proteins can be more advantageous, and pseudogenizing mutations might then be favored and increase in frequency, eventually becoming fixed in a species.

In humans, one of the examples that potentially supports the adaptive pseudogenization hypothesis is the MYH16 gene, for which inactivation has been suggested to be involved in human brain expansion (16). The loss of human CMAH gene function appears to be associated with the evolution of susceptibilities to malaria or typhoid toxins $(17,18)$. Loss of regulatory elements, as evidenced by hCONDEL.569 and HACNS1, can dramatically change expression patterns of nearby genes and confer human-specific anatomical traits $(19,20)$. In addition, a large number of human-specific gene losses have been identified thus far using comparative genomics studies (21-27). Here, we review representative cases of human gene inactivation and their functional implications.

\section{LOSS OF THE CMAH GENE AND SUSCEPTIBILITIES TO PATHOGENS}

The CMAH gene encodes for cytidine monophosphate- $\mathrm{N}$-acetylneuraminic acid hydroxylase, which is an enzyme responsible for the biosynthesis of $\mathrm{N}$-glycolylneuraminic acid (Neu5Gc), a hydroxylated form of the common sialic acid $\mathrm{N}$-acetylneuraminic acid (Neu5Ac) (28). Sequence comparisons have shown that there is a 92-bp deletion in the coding region of the human gene, while the chimpanzee gene is intact, indicating the human gene was inactivated after the human-chimpanzee divergence (29). The absence of active CMAH enzyme in humans resulted in differences in the glycan composition between humans and other primates: human sialoglycans terminate in Neu5Ac, whereas those of other primates and most other mammals terminate in Neu5Gc (30). The human-specific 
pseudogenization of the $\mathrm{CMAH}$ gene occurred approximately 3 MYA through an Alu-mediated exon deletion (31-33).

The phenotypic consequences of the loss of $\mathrm{CMAH}$ are of great interest and have been studied extensively. Initially, it was proposed that the loss of Neu5Gc moiety might be associated with the brain expansion of humans (32). However, a study has suggested that the differences in sialoglycan might instead be associated with the differences in malaria susceptibilities between humans and chimpanzees (34). Plasmodium falciparum is the global cause of malaria mortality in humans; however, it does not cause severe infection in chimpanzees. $P$. reichenowi, which is the closest relative of $P$. falciparum, infects chimpanzees and gorillas but not humans.

To explain this interesting relationship, the inactivation of the $C M A H$ gene in early humans and the subsequent evolution of the parasite has been proposed $(17,35)$. Inactivation of the $\mathrm{CMAH}$ gene rendered human ancestors incapable of synthesizing Neu5Gc from Neu5Ac. Abolishment of Neu5Gc from human erythrocytes might make humans resistant to $P$. reichenowi, which strongly prefers Neu5Gc. The resistance to $P$. reichenowi malaria could have been advantageous to human ancestors, and the inactive CMAH gene was fixed in the human lineage. More recently, however, a lineage of $P$. falciparum that is widely present in African great apes, especially in western gorillas, might have cross-infected humans (36). The strong preference for the overabundant Neu5Ac in human red blood cells might be responsible for the cross-species transmission of $P$. falciparum malaria and its emergence as the deadliest human pathogen $(17,34)$.

Glycans that terminate in Neu5Ac are associated with the host specificity of other exclusively human pathogens, such as Salmonella Typhi and human influenza A virus (IAV) $(18,37)$. The typhoid toxin selectively binds to Neu5Ac-terminated glycans and displays selective toxicity toward cells expressing them. Ferrets have also lost the CMAH gene through a nine-exon deletion, which is shared by the Pinnipedia and Musteloidia members of Carnivora, and they exhibit susceptibility to human-adapted IAV strains. Thus, the evolution of resistance to a certain pathogen through CMAH gene inactivation and subsequent development of susceptibility to other parasites is a good example of the evolutionary arms race between hosts and parasites $(35,38)$.

\section{LOSS OF THE MYH16 GENE AND REDUCTION OF MASTICATORY MUSCLES AND BRAIN EXPANSION}

The MYH16 gene encodes a sarcomeric myosin heavy chain, which is a major component of masticatory (jaw-closing) muscles (39). The human MYH16 gene is a pseudogene and does not produce functional protein due to a two-nucleotide deletion in exon 18. This deletion occurred in the human lineage after the human-chimpanzee divergence and has been fixed in modern humans (16). The loss of the MYH16 gene product in humans has been proposed to be associated with a marked re- duction in masticatory muscle mass, which might have allowed humans to have bigger brains $(16,40,41)$. Initially, this frameshift mutation in MYH16 was estimated to have appeared approximately 2.4 MYA, predating the appearance of Homo erectus/ergaster, which had a relatively gracile masticatory apparatus. The age of the inactivating mutation appears to support the idea that the loss of the MYH16 gene could be a crucial step for the enhanced encephalization of humans (16).

However, a more comprehensive analysis revealed that the human-specific deletion might have occurred approximately 5.3 MYA, which significantly precedes the first appearance of the genus Homo in the fossil record (42). The study also claimed that inactivation of the MYH16 gene would have had little contribution to the expansion of the brain, as the majority of brain growth in humans occurs long before the development of the masticatory musculature (43). Another study has indicated that, although humans have relatively small jaws and jaw muscles compared with those of closely related great apes, the human masticatory apparatus is highly efficient and can produce relatively high bite forces using low muscle forces (44).

It is possible that a dietary shift, perhaps to consuming softer foods, might have permitted smaller jaws in early humans, which led to a lower dependency on the MYH16 gene product (45). It is also likely that human ancestors evolved smaller jaws and chewing muscles without losing overall masticatory function. As a result, the MYH16 gene might have simply become extraneous, and under relaxed selection pressure, the gene accumulated disruptive mutations. Although the pseudogenization event of the MYH16 gene might not have directly driven encephalization, it is a compelling example of the association between gene inactivation and the acquisition of human-specific phenotypes.

\section{LOSS OF BITTER TASTE RECEPTOR GENES AND DIERTAY EVOLUTION}

There are five major taste sensations in humans and most other vertebrates: salty, sour, sweet, umami (savory), and bitter. Each of these tastes is perceived by distinct sets of taste receptors (46). In various mammals, taste receptor genes are often pseudogenized as they adapt to different dietary habits and life styles. For example, cats are not able to detect the sweetness of sugars because of their loss of functional sweet receptors, likely a result of their carnivorous behavior (47). Giant pandas lack a functional umami taste receptor gene; the inactivation of which was reported to coincide with their dietary shift to bamboo (48). Bottlenose dolphins lost receptors for the three basic tastes, sweet, bitter, and umami, most likely as a result of swallowing food whole, without chewing (49).

Bitter taste sensation is mediated by type 2 taste receptors (TAS2Rs) and is usually associated with detection and avoidance of toxic chemical substances or putrid food (46). Sequence comparison of TAS2R genes among mammals re- 
vealed that two of them, TAS2R62 and TAS2R64, are pseudogenes in humans, which became inactivated due to nonsense and/or frameshift mutations after the human-chimpanzee divergence and fixed in the modern human population $(50,51)$. These two pseudogenes are shared with other archaic humans, Neanderthals and Denisovans, indicating their ancient origins (52). TAS2R genes with an intact coding region also show relaxation of selective constraints, implying that the bitter taste sensation is generally reduced in the human lineage in comparison with other mammals (53).

Loss and/or relaxed selection of TAS2R genes may be associated with the dietary shift of ancestral humans (52). Human ancestors consumed more starch-rich tuberous roots such as yams, which generally tasted bitter. Extra calories obtained from these bitter root vegetables may have allowed humans to develop bigger brains. Eventually, humans learned to cook in order to remove bitter substances, which might have further reduced selection pressure on bitter taste receptor genes. Therefore, loss and/or relaxed selection of TAS2R genes might be deeply interwoven with the evolution of human dietary habits.

\section{LOSS OF OLFACTION-RELATED GENES AND EVOLUTION OF CHEMICAL SENSING AND SIGNALING}

Olfaction, or the perception of smell, is a crucial sense for animals and plays an important role in avoiding predators, searching for foods, and recognizing the opposite sex. Olfactory receptors (ORs) in the olfactory epithelium are responsible for the detection and discrimination of various odorants (54). The olfactory perception capabilities of humans, other apes, and Old World monkeys (OWMs) are generally considered to be significantly diminished when compared with other mammals, based on observations that these species have relatively small olfactory apparatuses and high number of OR pseudogenes (55-57). It has been proposed that humans and other catarrhines (OWMs and apes) have become more dependent on vision rather than olfaction, which created a relaxed selection pressure on OR genes (57).

However, various studies have suggested that there is no direct link between the evolution of trichromatic color vision and the degeneration of OR genes in catarrhines $(58,59)$. Another study claimed that humans are capable of distinguishing between more than 1 trillion olfactory stimuli (60). Recently, this estimation has been questioned and should be scrutinized by further studies (61).

Interestingly, some olfaction-related genes other than OR genes are inactivated in humans. For example, transient receptor potential cation channel, subfamily C, member 2 (TRPC2), which is required for pheromone sensing, is a pseudogene in humans $(62,63)$. A comparative genomics study of primates revealed that the TRPC2 gene is a pseudogene not only in humans but also in other catarrhines, indicating that the gene became inactivated in a common ancestor of OWMs and apes
(64). It has been speculated that the development of trichromatic color vision might have led to a reduced dependency on chemosensory communication for mediating a variety of social behaviors, although humans seem to still rely on chemosignals for certain interactions (65).

The MOXD2 gene, which encodes a highly conserved monooxygenase DBH-like 2 protein in vertebrates, is another inactive gene that is proposed to be involved in olfaction in humans (21). The human MOXD2 gene has an exon-deletion mutation, which occurred after the human-chimpanzee divergence. The mouse ortholog Moxd2 gene has been reported to be highly expressed in olfactory epithelium, implying that vertebrate MOXD2 could be involved in olfactory function (66). MOXD2 and its paralogs, MOXD1 and DBH, belong to the copper type II, ascorbate-dependent monooxygenase family. $\mathrm{DBH}$ is a dopamine $\beta$-hydroxylase, which converts dopamine to norepinephrine (noradrenaline) in the synaptic vesicles of postganglionic sympathetic neurons and for which deficiency or polymorphism is associated with various neuropsychiatric disorders (67-69). Vertebrate MOXD2 might also be involved in metabolism of neurotransmitters, potentially during transduction of olfactory stimuli.

The MOXD2 gene is also inactive in other apes: orangutans have multiple nonsense mutations and gibbons do not have the gene due to a genomic deletion that occurred in a common ancestor of all contemporary gibbons $(21,70)$. The gorilla gene shows an elevated non-synonymous substitution rate/synonymous substitution rate ratio, perhaps because its selection pressure has been recently relaxed, while the chimpanzee gene appears to be under purifying selection. Therefore, the loss of MOXD2 enzyme function might be associated with the reduced olfactory capabilities of humans and other apes. However, it remains uncertain whether this gene inactivation caused the diminished olfaction or the reduced dependency on olfaction led to relaxed selection pressure on the gene (21).

Interestingly, MOXD2 gene inactivation also occurred in another mammalian clade, the Cetacea (70). The dolphin and whale MOXD2 genes underwent a broad range of disruptive mutations, including nonsense, frameshift, and complete deletion. In whales, the TRPC2 gene is also a pseudogene, and many OR genes are not functional (71-73). The degeneration of these genes may coincide with the evolution of a fully aquatic lifestyle and highly sophisticated vocal communication and/or echolocation. Therefore, inactivation of olfaction-related genes of humans and other organisms may be a remarkable molecular signature of adaptive evolution to habitat shifts and/or sociobehavioral changes.

\section{POLYMORPHIC GENE INACTIVATION AND ASSOCIATED PHENOTYPES IN THE HUMAN POPULATION}

Sequence polymorphisms resulting in loss of function of a derived allele are frequently observed in the human population, 
indicating that gene inactivation events are rather common $(24,26,74-76)$. Population genetic studies have demonstrated that inactive allele frequencies range from rare to common, with some being nearly fixed $(24,26)$. Many of these inactive alleles have been reported to be associated with beneficial phenotypes in the individuals harboring them (75). Examples include: polymorphic pseudogenes in some TAS2R genes for bitter taste (51), a 32-bp deletion allele of the CCR5 gene that is found in relatively high frequency in Europeans (77), a nonsense allele of the CASP12 gene that is rare in Africans but very common in non-Africans $(78,79)$, and a nonsense allele of the ACTN3 gene that is commonly detected in various human populations (80).

Humans generally have 26 intact TAS2R genes for bitter taste sensation (51). TAS2R genes exhibit a high level of polymorphisms among human individuals, including non-synonymous substitutions and copy-number variations. Interestingly, some polymorphisms involve loss-of-function mutations; there are polymorphic pseudogenes in TAS2R2, TAS2R7, TAS2R45, and TAS2R46 as well as polymorphic whole-gene deletions in TAS2R43 and TAS2R45 (51, 53, 81-83). Loss of a specific $T A S 2 R$ gene is involved in individual-specific phenotypes in bitter taste sensation. For example, the bitter taste receptor encoded by the TAS2R43 gene responds to the artificial sweetener saccharin and contributes to the bitter aftertaste of saccharine and other related sweeteners (84). Lack of the TAS2R43 gene renders affected individuals to be insensitive to the bitterness of saccharine and other natural plant compounds, including aloin and aristolochic acid (83).

The human CCR5 gene encodes for C-C chemokine receptor type 5 (also known as CD195), which is a G-protein coupled receptor on the cell surface of white blood cells and acts as a receptor for chemokines (85). Human immunodeficiency viruses (HIVs) initially bind CCR5 proteins to enter and infect mucosal $\mathrm{CD}^{+} \mathrm{T}$ cells, which eventually may cause acquired immune deficiency syndrome (AIDS) (86, 87). A 32-bp deletion mutation in the CCR5 gene, referred to as the CCR5- 432 allele, is almost exclusively found in approximately $5 \%-14 \%$ of the European population and their descendants (77). The mutant allele produces defective proteins that cannot be detected on the cell surface. This lack of CCR5 protein on the cell surface protects individuals homozygous for the CCR5-432 allele from HIV infection (88). Because the frequency of this pseudogene allele is relatively high in Europeans, it was initially suggested that this allele appeared recently (approximately 1,000 years ago) and has undergone positive selection (77). However, a detailed study of the allele age and pattern of genetic variation revealed that the CCR5- $\Delta 32$ allele may have arisen more than 5,000 years ago and was selected for another reason or neutrally evolved, indicating that its resistance to HIV was pre-adaptive (89). Nevertheless, the resistance to HIVs and protection against AIDS as a result of CCR5 gene inactivation suggest that the CCR5 protein is a promising therapeutic target for preventing the spread of $\operatorname{HIV}(90,91)$.
The CASP12 gene encodes caspase 12, which belongs to a family of cysteine proteases that cleaves their substrates at C-terminal aspartic acid residues (92). There is a nonsense polymorphism in the human CASP12 gene, namely, a stop codon in exon 4 induces premature termination $(79,93)$. The inactive allele is very common in non-Africans, but rare in Africans. Intact full-length caspase 12 attenuates the inflammatory and innate immune responses to endotoxins, which can result in a severe septic response (79). Thus, the nonsense allele is advantageous as it confers resistance to severe sepsis, and it could have recently undergone positive selection in non-Africans $(25,78)$. The inactive allele appears to have originated in Africa and was initially neutral or approximately neutral. As human population size and density increased, individuals may have experienced more infectious diseases, for which the inactive allele was highly advantageous as a result of sepsis resistance (78). The human CASP12 gene is another example of a pre-adaptive gene inactivation and subsequent positive selection.

The human ACTN3 gene encodes $\alpha$-actinin-3, an actinbinding protein found in skeletal muscle (94). ACTN3 proteins are a major structural component of $Z$ lines and regulate the function of fast twitch (type II) muscle fibers, which underlie forceful and rapid muscle contraction during athletic activities such as sprinting (95). There is a nonsense polymorphism R577X in exon 16 of the ACTN3 gene $(80,95)$. This mutation results in undetectable ACTN3 protein in skeletal muscle. Interestingly, the ACTN3 genotype was suggested to be associated with human elite athletic performance; the $577 \mathrm{X}$ allele is associated with endurance, while the $577 \mathrm{R}$ allele is associated with sprinting and strength performance (96-98). The high frequency of the nonsense allele in human populations could have resulted from positive selection for improved endurance-running capabilities. This might have bestowed human ancestors increased opportunities for successful scavenging and/or persistence hunting (99-101).

Studies of human genomes have revealed a large number of loss-of-function mutations in humans that are polymorphic or fixed in populations. For example, a study on the genomes of Icelanders revealed a total of 6,795 autosomal loss-of-function alleles that are affected by nonsense or insertion/deletion mutations in 4,924 genes $(24,102)$. Some inactive alleles were found to be shared with archaic humans such as Neanderthals and are thought to be acquired by introgression (102). These loss-of-function mutations may contribute to the phenotypic variety of modern humans and could act as sources for adaptive evolution during various environmental changes (76).

\section{CONCLUSIONS}

The emergence of loss-of-function mutations and their subsequent expansion in modern humans indicate that gene inactivation is one of the mechanisms that confer novel advantageous phenotypes. A large number of gene inactivation events 
have been identified thus far in the human genome. Several of these exhibited strong association with beneficial traits. However, many have been neglected since these genes are nonfunctional pseudogenes in humans. Molecular functional studies of these genes in model organisms will provide clues about the molecular mechanisms for the emergence of human-specific phenotypes.

\section{ACKNOWLEDGEMENTS}

This study was supported by the National Research Foundation of Korea (NRF) grant (NRF-2012R1A1B3001513) funded by the Ministry of Education, Science and Technology, Republic of Korea.

\section{REFERENCES}

1. Chen FC and Li WH (2001) Genomic divergences between humans and other hominoids and the effective population size of the common ancestor of humans and chimpanzees. Am J Hum Genet 68, 444-456

2. Varki A and Altheide TK (2005) Comparing the human and chimpanzee genomes: searching for needles in a haystack. Genome Res 15, 1746-1758

3. Patterson N, Richter DJ, Gnerre S, Lander ES and Reich D (2006) Genetic evidence for complex speciation of humans and chimpanzees. Nature 441, 1103-1108

4. Enard W, Przeworski M, Fisher SE et al (2002) Molecular evolution of FOXP2, a gene involved in speech and language. Nature 418, 869-872

5. Konopka G, Bomar JM, Winden K et al (2009) Humanspecific transcriptional regulation of CNS development genes by FOXP2. Nature 462, 213-217

6. Pollard KS, Salama SR, Lambert N et al (2006) An RNA gene expressed during cortical development evolved rapidly in humans. Nature 443, 167-172

7. Beniaminov A, Westhof E and Krol A (2008) Distinctive structures between chimpanzee and human in a brain noncoding RNA. RNA 14, 1270-1275

8. Kim DS and Hahn Y (2011) Identification of human-specific transcript variants induced by DNA insertions in the human genome. Bioinformatics 27, 14-21

9. Kim DS and Hahn Y (2011) Identification of novel phosphorylation modification sites in human proteins that originated after the human-chimpanzee divergence. Bioinformatics 27, 2494-2501

10. Kim DS and Hahn Y (2012) Gains of ubiquitylation sites in highly conserved proteins in the human lineage. BMC Bioinformatics 13, 306

11. Kim DS and Hahn Y (2012) Human-specific protein isoforms produced by novel splice sites in the human genome after the human-chimpanzee divergence. BMC Bioinformatics 13, 299

12. Kim DS and Hahn Y (2015) The acquisition of novel $\mathrm{N}$-glycosylation sites in conserved proteins during human evolution. BMC Bioinformatics 16, 29

13. Prabhakar S, Visel A, Akiyama JA et al (2008) Humanspecific gain of function in a developmental enhancer.
Science 321, 1346-1350

14. Rogers J and Gibbs RA (2014) Comparative primate genomics: emerging patterns of genome content and dynamics. Nat Rev Genet 15, 347-359

15. Olson MV (1999) When less is more: gene loss as an engine of evolutionary change. Am J Hum Genet 64, 18-23

16. Stedman HH, Kozyak BW, Nelson A et al (2004) Myosin gene mutation correlates with anatomical changes in the human lineage. Nature 428, 415-418

17. Rich SM, Leendertz FH, Xu G et al (2009) The origin of malignant malaria. Proc Natl Acad Sci U S A 106, 14902-14907

18. Deng L, Song J, Gao X et al (2014) Host adaptation of a bacterial toxin from the human pathogen Salmonella Typhi. Cell 159, 1290-1299

19. McLean CY, Reno PL, Pollen AA et al (2011) Humanspecific loss of regulatory DNA and the evolution of human-specific traits. Nature 471, 216-219

20. Sumiyama K and Saitou N (2011) Loss-of-function mutation in a repressor module of human-specifically activated enhancer HACNS1. Mol Biol Evol 28, 3005-3007

21. Hahn $Y$, Jeong $S$ and Lee B (2007) Inactivation of MOXD2 and S100A15A by exon deletion during human evolution. Mol Biol Evol 24, 2203-2212

22. Hahn $Y$ and Lee B (2005) Identification of nine humanspecific frameshift mutations by comparative analysis of the human and the chimpanzee genome sequences. Bioinformatics 21 Suppl 1, i186-194

23. Hahn $Y$ and Lee B (2006) Human-specific nonsense mutations identified by genome sequence comparisons. Hum Genet 119, 169-178

24. Sulem $P$, Helgason $H$, Oddson A et al (2015) Identification of a large set of rare complete human knockouts. Nat Genet 47, 448-452

25. Wang X, Grus WE and Zhang J (2006) Gene losses during human origins. PLoS Biol 4, e52

26. Yngvadottir B, Xue Y, Searle S et al (2009) A genome-wide survey of the prevalence and evolutionary forces acting on human nonsense SNPs. Am J Hum Genet 84, 224-234

27. Zhu J, Sanborn JZ, Diekhans M, Lowe CB, Pringle TH and Haussler D (2007) Comparative genomics search for losses of long-established genes on the human lineage. PLoS Comput Biol 3, e247

28. Bergfeld AK, Pearce OM, Diaz SL, Pham T and Varki A (2012) Metabolism of vertebrate amino sugars with $\mathrm{N}$-glycolyl groups: elucidating the intracellular fate of the non-human sialic acid $\mathrm{N}$-glycolylneuraminic acid. J Biol Chem 287, 28865-28881

29. Chou HH, Takematsu H, Diaz S et al (1998) A mutation in human CMP-sialic acid hydroxylase occurred after the Homo-Pan divergence. Proc Natl Acad Sci U S A 95, 11751-11756

30. Varki NM, Strobert E, Dick EJ Jr, Benirschke K and Varki A (2011) Biomedical differences between human and nonhuman hominids: potential roles for uniquely human aspects of sialic acid biology. Annu Rev Pathol 6, 365393

31. Hayakawa T, Satta $Y$, Gagneux P, Varki A and Takahata $N$ (2001) Alu-mediated inactivation of the human CMP- 
$\mathrm{N}$-acetylneuraminic acid hydroxylase gene. Proc Natl Acad Sci U S A 98, 11399-11404

32. Chou HH, Hayakawa T, Diaz S et al (2002) Inactivation of CMP-N-acetylneuraminic acid hydroxylase occurred prior to brain expansion during human evolution. Proc Natl Acad Sci U S A 99, 11736-11741

33. Hayakawa T, Aki I, Varki A, Satta $Y$ and Takahata $N$ (2006) Fixation of the human-specific CMP-N-acetylneuraminic acid hydroxylase pseudogene and implications of haplotype diversity for human evolution. Genetics 172, 1139-1146

34. Martin MJ, Rayner JC, Gagneux P, Barnwell JW and Varki A (2005) Evolution of human-chimpanzee differences in malaria susceptibility: relationship to human genetic loss of N-glycolylneuraminic acid. Proc Natl Acad Sci U S A 102, 12819-12824

35. Varki A and Gagneux P (2009) Human-specific evolution of sialic acid targets: explaining the malignant malaria mystery? Proc Natl Acad Sci U S A 106, 14739-14740

36. Liu W, Li Y, Learn GH et al (2010) Origin of the human malaria parasite Plasmodium falciparum in gorillas. Nature 467, 420-425

37. Takahashi T, Takano M, Kurebayashi $Y$ et al (2014) $\mathrm{N}$-glycolylneuraminic acid on human epithelial cells prevents entry of influenza A viruses that possess $\mathrm{N}$-glycoIylneuraminic acid binding ability. J Virol 88, 8445-8456

38. Dawkins R and Krebs JR (1979) Arms races between and within species. Proc R Soc Lond B Biol Sci 205, 489-511

39. Desjardins PR, Burkman JM, Shrager JB, Allmond LA and Stedman HH (2002) Evolutionary implications of three novel members of the human sarcomeric myosin heavy chain gene family. Mol Biol Evol 19, 375-393

40. Currie P (2004) Human genetics: muscling in on hominid evolution. Nature 428, 373-374

41. Pennisi E (2004) Human evolution. The primate bite: brawn versus brain? Science 303, 1957

42. Perry GH, Verrelli BC and Stone AC (2005) Comparative analyses reveal a complex history of molecular evolution for human MYH16. Mol Biol Evol 22, 379-382

43. McCollum MA, Sherwood CC, Vinyard CJ, Lovejoy CO and Schachat $F$ (2006) Of muscle-bound crania and human brain evolution: the story behind the MYH16 headlines. J Hum Evol 50, 232-236

44. Wroe S, Ferrara TL, McHenry CR, Curnoe D and Chamoli U (2010) The craniomandibular mechanics of being human. Proc Biol Sci 277, 3579-3586

45. Hoh JF (2002) 'Superfast' or masticatory myosin and the evolution of jaw-closing muscles of vertebrates. J Exp Biol 205, 2203-2210

46. Bachmanov AA and Beauchamp GK (2007) Taste receptor genes. Annu Rev Nutr 27, 389-414

47. Li X, Li W, Wang $\mathrm{H}$ et al (2005) Pseudogenization of a sweet-receptor gene accounts for cats' indifference toward sugar. PLoS Genet 1, 27-35

48. Zhao H, Yang JR, Xu H and Zhang J (2010) Pseudogenization of the umami taste receptor gene Tas1r1 in the giant panda coincided with its dietary switch to bamboo. Mol Biol Evol 27, 2669-2673

49. Jiang $\mathrm{P}$, Josue J, Li X et al (2012) Major taste loss in carnivorous mammals. Proc Natl Acad Sci U S A 109,
4956-4961

50. Parry CM, Erkner A and le Coutre J (2004) Divergence of $\mathrm{T} 2 \mathrm{R}$ chemosensory receptor families in humans, bonobos, and chimpanzees. Proc Natl Acad Sci U S A 101, 14830-14834

51. Hayakawa T, Suzuki-Hashido N, Matsui A and Go $Y$ (2014) Frequent expansions of the bitter taste receptor gene repertoire during evolution of mammals in the Euarchontoglires clade. Mol Biol Evol 31, 2018-2031

52. Perry GH, Kistler L, Kelaita MA and Sams AJ (2015) Insights into hominin phenotypic and dietary evolution from ancient DNA sequence data. J Hum Evol 79, 55-63

53. Wang X, Thomas SD and Zhang J (2004) Relaxation of selective constraint and loss of function in the evolution of human bitter taste receptor genes. Hum Mol Genet 13, 2671-2678

54. Buck $L$ and Axel R (1991) A novel multigene family may encode odorant receptors: a molecular basis for odor recognition. Cell 65, 175-187

55. Smith TD and Bhatnagar KP (2004) Microsmatic primates: reconsidering how and when size matters. Anat Rec B New Anat 279, 24-31

56. Dong D, He G, Zhang S and Zhang Z (2009) Evolution of olfactory receptor genes in primates dominated by birth-and-death process. Genome Biol Evol 1, 258-264

57. Barton RA (2006) Olfactory evolution and behavioral ecology in primates. Am J Primatol 68, 545-558

58. Niimura $Y$ (2012) Olfactory receptor multigene family in vertebrates: from the viewpoint of evolutionary genomics. Curr Genomics 13, 103-114

59. Matsui A, Go Y and Niimura Y (2010) Degeneration of olfactory receptor gene repertories in primates: no direct link to full trichromatic vision. Mol Biol Evol 27, 11921200

60. Bushdid C, Magnasco MO, Vosshall LB and Keller A (2014) Humans can discriminate more than 1 trillion olfactory stimuli. Science 343, 1370-1372

61. Gerkin RC and Castro JB (2015) Humans can discriminate trillions of olfactory stimuli, or more, or fewer. arXiv 1502, 05120

62. Kiselyov K, van Rossum DB and Patterson RL (2010) TRPC channels in pheromone sensing. Vitam Horm 83, 197-213

63. Vannier B, Peyton M, Boulay G et al (1999) Mouse trp2, the homologue of the human trpc2 pseudogene, encodes mTrp2, a store depletion-activated capacitative $\mathrm{Ca}^{2+}$ entry channel. Proc Natl Acad Sci U S A 96, 2060-2064

64. Liman ER and Innan H (2003) Relaxed selective pressure on an essential component of pheromone transduction in primate evolution. Proc Natl Acad Sci U S A 100, 3328-3332

65. Lubke KT and Pause BM (2015) Always follow your nose: the functional significance of social chemosignals in human reproduction and survival. Horm Behav 68, 134-144

66. Su Al, Wiltshire T, Batalov S et al (2004) A gene atlas of the mouse and human protein-encoding transcriptomes. Proc Natl Acad Sci U S A 101, 6062-6067

67. Timmers HJ, Deinum J, Wevers RA and Lenders JW (2004) Congenital dopamine- $\beta$-hydroxylase deficiency in 
humans. Ann N Y Acad Sci 1018, 520-523

68. Cubells JF, Sun X, Li W et al (2011) Linkage analysis of plasma dopamine $\beta$-hydroxylase activity in families of patients with schizophrenia. Hum Genet 130, 635-643

69. Combarros O, Warden DR, Hammond N et al (2010) The dopamine $\beta$-hydroxylase $-1021 \mathrm{C} / \mathrm{T}$ polymorphism is associated with the risk of Alzheimer's disease in the Epistasis Project. BMC Med Genet 11, 162

70. Kim DS, Wang Y, Oh HJ, Lee K and Hahn Y (2014) Frequent loss and alteration of the MOXD2 gene in catarrhines and whales: a possible connection with the evolution of olfaction. PLoS One 9, e104085

71. Yu L, Jin W, Wang JX et al (2010) Characterization of TRPC2, an essential genetic component of VNS chemoreception, provides insights into the evolution of pheromonal olfaction in secondary-adapted marine mammals. Mol Biol Evol 27, 1467-1477

72. Yim HS, Cho YS, Guang X et al (2014) Minke whale genome and aquatic adaptation in cetaceans. Nat Genet 46, 88-92

73. McGowen MR, Clark C and Gatesy J (2008) The vestigial olfactory receptor subgenome of odontocete whales: phylogenetic congruence between gene-tree reconciliation and supermatrix methods. Syst Biol 57, 574-590

74. MacArthur DG and Tyler-Smith C (2010) Loss-of-function variants in the genomes of healthy humans. Hum Mol Genet 19, R125-130

75. Kaiser J (2014) The hunt for missing genes. Science 344, 687-689

76. Alkuraya FS (2015) Human knockout research: new horizons and opportunities. Trends Genet 31, 108-115

77. Stephens JC, Reich DE, Goldstein DB et al (1998) Dating the origin of the CCR5-D32 AIDS-resistance allele by the coalescence of haplotypes. Am J Hum Genet 62, 1507-1515

78. Xue Y, Daly A, Yngvadottir B et al (2006) Spread of an inactive form of caspase-12 in humans is due to recent positive selection. Am J Hum Genet 78, 659-670

79. Saleh M, Vaillancourt JP, Graham RK et al (2004) Differential modulation of endotoxin responsiveness by human caspase-12 polymorphisms. Nature 429, 75-79

80. North KN, Yang N, Wattanasirichaigoon D, Mills $M$, Easteal S and Beggs AH (1999) A common nonsense mutation results in a-actinin-3 deficiency in the general population. Nat Genet 21, 353-354

81. Go Y, Satta Y, Takenaka O and Takahata N (2005) Lineage-specific loss of function of bitter taste receptor genes in humans and nonhuman primates. Genetics 170, 313-326

82. Kim U, Wooding S, Ricci D, Jorde LB and Drayna D (2005) Worldwide haplotype diversity and coding sequence variation at human bitter taste receptor loci. Hum Mutat 26, 199-204

83. Pronin AN, Xu H, Tang H, Zhang L, Li Q and Li X (2007) Specific alleles of bitter receptor genes influence human sensitivity to the bitterness of aloin and saccharin. Curr Biol 17, 1403-1408

84. Roudnitzky N, Bufe B, Thalmann S et al (2011) Genomic, genetic and functional dissection of bitter taste responses to artificial sweeteners. Hum Mol Genet 20,
3437-3449

85. Samson $M$, Labbe $O$, Mollereau $C$, Vassart $G$ and Parmentier M (1996) Molecular cloning and functional expression of a new human CC-chemokine receptor gene. Biochemistry 35, 3362-3367

86. Dragic T, Litwin V, Allaway GP et al (1996) HIV-1 entry into $\mathrm{CD}^{+}$cells is mediated by the chemokine receptor CC-CKR-5. Nature 381, 667-673

87. Deng H, Liu R, Ellmeier W et al (1996) Identification of a major co-receptor for primary isolates of HIV-1. Nature 381, 661-666

88. Liu R, Paxton WA, Choe S et al (1996) Homozygous defect in HIV-1 coreceptor accounts for resistance of some multiply-exposed individuals to HIV-1 infection. Cell 86, 367-377

89. Sabeti PC, Walsh E, Schaffner SF et al (2005) The case for selection at CCR5-D32. PLoS Biol 3, e378

90. Hutter G, Nowak D, Mossner M et al (2009) Long-term control of HIV by CCR5 Delta32/Delta32 stem-cell transplantation. N Engl J Med 360, 692-698

91. Tebas P, Stein D, Tang WW et al (2014) Gene editing of CCR5 in autologous CD4 T cells of persons infected with HIV. N Engl J Med 370, 901-910

92. Alnemri ES, Livingston DJ, Nicholson DW et al (1996) Human ICE/CED-3 protease nomenclature. Cell 87, 171

93. Fischer $\mathrm{H}$, Koenig $U$, Eckhart $L$ and Tschachler E (2002) Human caspase 12 has acquired deleterious mutations. Biochem Biophys Res Commun 293, 722-726

94. Beggs AH, Byers TJ, Knoll JH, Boyce FM, Bruns GA and Kunkel LM (1992) Cloning and characterization of two human skeletal muscle alpha-actinin genes located on chromosomes 1 and 11. J Biol Chem 267, 9281-9288

95. Mills M, Yang N, Weinberger R et al (2001) Differential expression of the actin-binding proteins, $\alpha$-actinin- 2 and -3 , in different species: implications for the evolution of functional redundancy. Hum Mol Genet 10, 1335-1346

96. Yang N, MacArthur DG, Gulbin JP et al (2003) ACTN3 genotype is associated with human elite athletic performance. Am J Hum Genet 73, 627-631

97. Niemi AK and Majamaa K (2005) Mitochondrial DNA and ACTN3 genotypes in Finnish elite endurance and sprint athletes. Eur J Hum Genet 13, 965-969

98. Roth SM, Walsh S, Liu D, Metter EJ, Ferrucci L and Hurley BF (2008) The ACTN3 R577X nonsense allele is under-represented in elite-level strength athletes. Eur J Hum Genet 16, 391-394

99. Bramble DM and Lieberman DE (2004) Endurance running and the evolution of Homo. Nature 432, 345-352

100. MacArthur DG, Seto JT, Raftery JM et al (2007) Loss of ACTN3 gene function alters mouse muscle metabolism and shows evidence of positive selection in humans. Nat Genet 39, 1261-1265

101. Ruxton GD and Wilkinson DM (2013) Endurance running and its relevance to scavenging by early hominins. Evolution 67, 861-867

102. Lin YL, Pavlidis P, Karakoc E, Ajay J and Gokcumen O (2015) The evolution and functional impact of human deletion variants shared with archaic hominin genomes. Mol Biol Evol 32, 1008-1019 